\title{
Effect of feed supplementation with L-carnitine on growth and cold tolerance of the Nile tilapia, Oreochromis niloticus
}

\author{
Hosam, M. Agouz'; Magdy, A. Soltan' ${ }^{2}$ Abd-Elkarem, E. Esaid ${ }^{2}$; and \\ Abd-Elbary, A. El-Qabatti ${ }^{2}$ \\ 1- Central lab of aquaculture research, Abassa, Abou-Hammad, Sharkia, Egypt. \\ 2- Department of Animal production, Faculty of Agriculture, Benha University. Egypt.
}

\section{ABSTRACT}

The effect of dietary L-carnitine and lipid on growth performance, feed utilization and body composition of Nile tilapia, Oreochromis niloticus were evaluated in two separate $2 \times 5$ factorial experiments as well as the effect of dietary L-carnitine on cold tolerance of Nile tilapia was evaluated in another experiment of the present study. The effect of L-carnitine was evaluated in five levels, $0,300,600,900$ and $1200 \mathrm{mg} / \mathrm{kg}$ diet at each of two lipid levels (5 and $10 \%)$, therefore ten isonitrogenous $(30 \% \mathrm{CP}$ ) and isocaloric $(3000 \mathrm{kcal} \mathrm{ME} / \mathrm{kg})$ diets were formulated and tested in three replicates with Nile tilapia (First experiment). Another experiment was applied by using the same previous diets $(30 \% \mathrm{CP}, 5 \%$ fat, $3000 \mathrm{kcal} \mathrm{ME} / \mathrm{kg}$ diet) which formulated to contain increasing levels of L-carnitine, 0, 300, 600, 900 and 1200 $\mathrm{mg} / \mathrm{kg}$ diet to study the effect of L-carnitine on cold tolerance of Nile tilapia (second experiment).

In the present study, dietary L-carnitine at any level significantly increased all growth parameters (body weight, body length, weight gain, specific growth rate) and improved feed conversion and protein efficiency ratio of Nile tilapia. Compared to control group dietary Lcarnitine supplementation significantly $(\mathrm{P}<0.05)$ increased the percentage of dress-out and flesh and decreased the percentage of by-products of Nile tilapia Also, increased dietary L-carnitine level decreased $(\mathrm{P}<0.05)$ fat content and increased $(\mathrm{P}<0.05)$ protein content of Nile tilapia.

Results of the second experiment of the present study clearly showed that, the addition of L-carnitine to the diet of tilapia fish can substantially reduce their mortality during winter season. Fish fed the diet supplemented with 900 or $1200 \mathrm{mg} / \mathrm{kg}$ exhibited the best tolerance to cold water.

In conclusion, dietary L-carnitine supplementation at all studied levels (300 to 1200 $\mathrm{mg} / \mathrm{kg}$ diet) improved growth and feed utilization, decrease tissue fat and increased tissue protein and also increased survival rate of tilapia fry reared during cold season.

Keywords: L-carnitine, lipids, growth performance, feed utilization Nile tilapia

\section{INTRODUCTION}

L-carnitine ( $\gamma$ - trimethyl-amino- $\beta$-hydroxybutyrate) is synthesized in vivo from lysine and methionine and is essential for the transport of long-chain fatty acids from the cytosol into the mitochondria where the $\beta$-oxidation of these fatty acids occurs (Dunn, 1981). Fish biologists first became interested in L-carnitine when Bilinski and Jonas (1970) observed that addition of L-carnitine to their incubation media enhanced transport and oxidation of long chain fatty acids in isolated trout mitochondria.

The improved energy production in mitochondria through B-oxidation of fatty acids may be suggest that exogenous administration of L-carnitine could enhance the performance of fish by improving energy utilization efficiency from lipid oxidation (Torreele et al., 1993; Chatzifotis et al., 1995).

It has also been found that there is an increased tolerance of ammonia (Tremblay and Bradley 1992) that can not be directly explained by the effect of L-carnitine. It also increases the rate of protein synthesis (Santulli et al., 1990) and enhancing the generation of metabolic energy. 
This could stimulate some specific cell functions and may influence several biochemical and physiological process, i.e., cell protection against xenobiotics (Torreele et al., 1993; Chatzifotis et al., 1995).

Lipid nutrition of fish produced in aquaculture has attracted considerable interest both historically from the standpoint of satisfying essential fatty acid requirements (NRC, 1993) and more recently for the protein-sparing capability. Most warmwater species are typically fed diets with less than $10 \%$ lipid due to their ability to utilize higher levels of carbohydrates as energy source, the high cost of lipid relative to carbohydrates, and unwanted accretion of lipid depots (Soltan et al., $2002 \&$ 2006). To improve utilization of dietary lipids by fish, L-carnitine has shown promise by improving growth and feed efficiency and reducing lipid deposition in some fish species (Torreele et al., 1993; Chatzifotis et al., 1995 and Ji et al., 1996)

Chichlids such as tilapia, are amongst the most popular and promising fish for warm water aquaculture and/or ornamental fish production. One of the most serious drawbacks to growing this specie in temperate zones is their sensitivity to low ambient temperature, leading in extreme cold temperature to mass mortality (Soltan et $a l ., 2015)$. Overwintering therefore presents a serious problem. Therefore, the purpose of the present study was to determine if Nile tilapia could utilize elevated levels of dietary lipid, and if supplemental L-carnitine could enhance utilization of dietary lipid. Also the effect of dietary L-carnitine on cold tolerance of Nile tilapia was evaluated.

\section{MATERIALS AND METHODS}

The experimental work of the present study was carried out at Fish Nutrition Laboratory, Faculty of Agriculture, Benha University.

\section{Growth performance experiments:}

Fish: Nile tilapia, O. niloticus fingerlings were obtained from Abbassa hatchery, Sharkia Governorate. The experimental fish were transported in a 50-liter plastic bags filled with water and oxygen to the fish laboratory. Fish were adapted for two weeks and then distributed randomly into twenty glass aquaria $(100 \times 40 \times 50 \mathrm{~cm})$. Each fish was taken out by a net and weighed then transferred randomly to the experimental aquaria. Twenty tanks were randomly stocked with 30 tilapia fish for each tank (first experiment).

Fish grouping: For each experiment fish were grouped into control group (0 Lcarnitine) and four dietary L-carnitine (Arab Company For Pharmaceuticals \& Medical Plants - MEPACO - Egypt) at concentrations of 300, 600, 900 and 1200 $\mathrm{mg} / \mathrm{kg}$ diet in two dietary lipid levels, 5 and $10 \%$. Therefore, the experimental diets designated as F5C0, F5C300, F5C600, F5C900, F5C1200, F10C0, F10C300, F10C600, F10C900 and F10C1200, respectively. All these fish groups were arranged in two replicates.

Feed and feeding: Composition and proximate analysis of basal diets used in the two experiments are presented in Table 1. Two basal diets were formulated to contain 5 or $10 \%$ dietary lipid. Each basal diet was divided into 5 diets and L-carnitine was added with five levels, $0,300,600,900$ and $1200 \mathrm{mg} / \mathrm{kg}$ diet, therefore 10 isonitrogenous (30\% CP) and isocaloric $(3000 \mathrm{kcal} \mathrm{ME} / \mathrm{kg}$ ) diets were formulated. Fish were given the diets at a daily rate 5\% of total biomass. Fish were fed twice daily at 9:00 am and 2:00 pm. Records of live body weight $(\mathrm{g})$ and body length $(\mathrm{cm})$ of individual fish were measured at the start and the end of each experiment. Growth performance and feed utilization parameters were measured by using the following equations: 
Specific growth rate $(\mathrm{SGR})=\frac{\mathrm{LnW} 2-\mathrm{LnW} 1}{\mathrm{t}} \times 100$

Where:- $\mathrm{Ln}=$ the natural $\log , \mathrm{W} 1=$ initial fish weight; $\mathrm{W} 2=$ the final fish weight in "grams" and $\mathrm{t}=$ period in days.

Weight gain $(\mathrm{WG})=$ final weight $(\mathrm{g})$ - initial weight $(\mathrm{g})$

Feed conversion ratio $(\mathrm{FCR})=$ feed ingested $(\mathrm{g}) /$ weight gain $(\mathrm{g})$

Protein efficiency ratio $($ PER $)=$ weight gain $(\mathrm{g}) /$ protein ingested $(\mathrm{g})$

At the end of each experiment, three fish were randomly sampled from each aquarium and slaughtered. The weight of head, viscera, flesh, carcass and total byproducts were recorded. All carcass components were measured according to Lovell (1981). Another three fish were also chosen at random and exposed to the proximate analysis of whole fish body according to the methods of AOAC (1990).

\section{Cold tolerance experiment:}

The second experiment of the present study aimed to study the effect of dietary L-carnitine on cold tolerance of Nile tilapia, therefore, 10 glass aquaria $(100 \times 40 \times 50$ $\mathrm{cm}$ ) were stocked with 40 tilapia for each aquarium and the aquaria were divided into five groups (in two replicates). During the experimental period (from 1December to 1 March 2004) fish were fed five isonitrogenous (30\% CP) and isocaloric (3000 ME/kg diet) diets at a rate of $1 \%$ of fish body weight ( 6 days/week). The experimental diets formulated to contains, $0,300,600,900$ and $1200 \mathrm{mg}$ L-carnitine $/ \mathrm{kg}$ diet. Temperature was weekly recorded in all aquaria.

Statistical analysis: Statistical analysis of the obtained data was analyzed according to SAS (1996). Differences between means were tested for significance according to Duncan's multiple rang test as described by Duncan (1955).

Table 1: Composition and proximate analysis of basal diets.

\begin{tabular}{|c|c|c|}
\hline Ingredient & $5 \%$ lipid & $10 \%$ lipid \\
\hline Fish meal & 28.0 & 28.0 \\
\hline Soybean meal & 18.0 & 18.0 \\
\hline Yellow corn & 24.0 & 16.5 \\
\hline Wheat flour & 13.0 & 9.0 \\
\hline Wheat bran & 9.0 & 15.5 \\
\hline Corn oil & 4.0 & 9.0 \\
\hline Vit.\&Min. mix. ${ }^{1}$ & 4.0 & 4.0 \\
\hline Sum & 100.0 & 100.0 \\
\hline \multicolumn{3}{|c|}{ Proximate analysis (Dry matter basis) } \\
\hline Dry matter & 95.34 & 95.78 \\
\hline Protein & 30.12 & 30.23 \\
\hline Lipid & 5.32 & 10.11 \\
\hline Ash & 7.96 & 8.11 \\
\hline Crude fiber & 9.87 & 9.55 \\
\hline $\mathrm{NFE}^{2}$ & 46.73 & 42.00 \\
\hline $\mathrm{ME}(\mathrm{Kcal} / \mathrm{kg} \operatorname{diet})^{3}$ & 3019 & 3028 \\
\hline $\mathrm{P} / \mathrm{E} \mathrm{ratio}^{4}$ & 99.77 & 99.83 \\
\hline
\end{tabular}

Vitamin \& mineral mixture/kg premix:: Vitamin D3, 0.8 million IU; A, 4.8 million IU; E, 4 g; K, 0.8 g, B1, 0.4 g; Riboflavin, 1.6 g; B6, 0.6 g, B12, 4 mg; Pantothenic acid, 4 g; Nicotinic acid, 8 g; Folic acid, 0.4 g Biotin, 20 mg , Mn, 22 g; Zn, 22 g; Fe, 12 g; Cu, 4 g; I, 0.4 g, Selenium, 0.4 g and Co, 4.8 mg.

${ }^{2}$ Nitrogen free extract $(\mathrm{NFE})=100-(\mathrm{CP}+\mathrm{EE}+\mathrm{CF}+\mathrm{Ash})$

${ }^{3}$ Metabolizable energy was calculated from ingredients based on NRC (1993) values for tilapia.

${ }^{4}$ Protein to energy ratio: $\mathrm{mg}$ protein/kcal ME. 


\section{RESULTS AND DISCUSSION}

\section{First experiment}

\section{I-Growth performance, feed intake and feed utilization}

Table 2 showed the effect of L-carnitine on growth performance and feed utilization of Nile tilapia, it revealed that, at the experiment start body weight (BW) ranged between 5.78 to $6.13 \mathrm{~g}$ and body length (BL) ranged between 6.90 to $7.27 \mathrm{~cm}$ with no significant differences between fish groups in BW and BL indicating the random distribution of fish around the different treatments. At the end of the experimental period ( 90 days) results of Table 2 indicated that, dietary factors had dramatic effects on growth and feed utilization. Dietary lipid levels studied (5 and $10 \%$ ) had no significant effect on all growth and feed utilization parameters while all dietary L-carnitine levels significantly $(\mathrm{P}<0.05)$ increased $\mathrm{BW}, \mathrm{BL}, \mathrm{WG}, \mathrm{SGR}$, feed intake (FI) and improved FCR and PER. The obtained results follow the trend as those obtained by Abdel-Hakim et al., (2001 a \& b); Hassaan et al., (2013 and 2014 a \& b).

Table 2: Least square means and standard error for the effect of dietary fat and L-carnitine on growth performance and feed utilization of Nile tilapia.

\begin{tabular}{|c|c|c|c|c|c|c|c|c|c|c|}
\hline \multirow[t]{2}{*}{ Item } & \multirow[t]{2}{*}{ No. } & \multicolumn{2}{|c|}{ Body weight (g) } & \multicolumn{2}{|c|}{$\begin{array}{l}\text { Body length } \\
(\mathrm{cm})\end{array}$} & \multirow{2}{*}{$\begin{array}{l}\text { Weight } \\
\text { gain } \\
\text { (WG) }\end{array}$} & \multirow{2}{*}{$\begin{array}{l}\text { Specific } \\
\text { growth } \\
\text { rate (SGR) }\end{array}$} & \multirow{2}{*}{$\begin{array}{l}\text { Feed } \\
\text { intake } \\
(\mathrm{g}) / \mathrm{fish} \\
(\mathrm{FI})\end{array}$} & \multirow{2}{*}{$\begin{array}{c}\text { Feed } \\
\text { conversion } \\
\text { ratio (FCR) }\end{array}$} & \multirow{2}{*}{$\begin{array}{c}\text { Protein } \\
\text { efficiency } \\
\text { ratio (PER) }\end{array}$} \\
\hline & & Initial & Final & Initial & Final & & & & & \\
\hline \multicolumn{11}{|l|}{ Fat $(\mathbf{F})$} \\
\hline $5 \%(\mathrm{~F} 5)$ & 300 & $\begin{array}{l}5.91 \pm \\
0.12\end{array}$ & $\begin{array}{c}24.59 \pm \\
0.31\end{array}$ & $\begin{array}{l}7.15 \pm \\
0.05\end{array}$ & $\begin{array}{c}11.51 \pm \\
0.06\end{array}$ & $\begin{array}{c}18.69 \pm \\
0.02\end{array}$ & $\begin{array}{l}1.69 \pm \\
0.01\end{array}$ & $\begin{array}{l}51.54 \pm \\
0.15\end{array}$ & $\begin{array}{l}2.76 \pm \\
0.02\end{array}$ & $\begin{array}{l}1.20 \pm \\
0.12\end{array}$ \\
\hline $10 \%(\mathrm{~F} 10)$ & 300 & $\begin{array}{c}6.04 \pm \\
0.12 \\
\end{array}$ & $\begin{array}{c}25.19 \pm \\
0.31 \\
\end{array}$ & $\begin{array}{l}7.12 \pm \\
0.05 \\
\end{array}$ & $\begin{array}{c}11.71 \pm \\
0.06 \\
\end{array}$ & $\begin{array}{c}19.15 \pm \\
0.02 \\
\end{array}$ & $\begin{array}{c}1.70 \pm \\
0.01 \\
\end{array}$ & $\begin{array}{c}52.59 \pm \\
0.15 \\
\end{array}$ & $\begin{array}{c}2.75 \pm \\
0.02 \\
\end{array}$ & $\begin{array}{c}1.21 \pm \\
0.12 \\
\end{array}$ \\
\hline \multicolumn{11}{|c|}{ L-carn. mg/kg diet ( C ) } \\
\hline $0(\mathrm{C} 0)$ & 120 & $\begin{array}{c}5.81 \pm \\
0.21 \\
\end{array}$ & $\begin{array}{l}19.83 \pm \\
0.54 \mathrm{~b} \\
\end{array}$ & $\begin{array}{l}7.11 \pm \\
0.09 \\
\end{array}$ & $\begin{array}{l}10.64 \pm \\
0.11 \mathrm{~b} \\
\end{array}$ & $\begin{array}{l}14.02 \pm \\
0.03 \mathrm{e} \\
\end{array}$ & $\begin{array}{l}1.47 \pm \\
0.02 \mathrm{~b} \\
\end{array}$ & $\begin{array}{l}43.64 \pm \\
0.25 \mathrm{~b} \\
\end{array}$ & $\begin{array}{l}3.12 \pm \\
0.03 \mathrm{a} \\
\end{array}$ & $\begin{array}{l}1.07 \pm \\
0.20 \mathrm{~b} \\
\end{array}$ \\
\hline $300(\mathrm{C} 300)$ & 120 & $\begin{array}{c}5.97 \pm \\
0.21\end{array}$ & $\begin{array}{c}25.64 \pm \\
0.54 \mathrm{a} \\
\end{array}$ & $\begin{array}{l}7.16 \pm \\
0.09 \\
\end{array}$ & $\begin{array}{c}11.98 \pm \\
0.11 \mathrm{a} \\
\end{array}$ & $\begin{array}{l}19.67 \pm \\
0.03 \mathrm{~d}\end{array}$ & $\begin{array}{l}1.74 \pm \\
0.02 \mathrm{a} \\
\end{array}$ & $\begin{array}{c}53.39 \pm \\
0.25 \mathrm{a} \\
\end{array}$ & $\begin{array}{l}2.71 \pm \\
0.03 \mathrm{~b}\end{array}$ & $\begin{array}{l}1.23 \pm \\
0.20 \mathrm{a} \\
\end{array}$ \\
\hline $600(\mathrm{C} 600)$ & 120 & $\begin{array}{c}5.98 \pm \\
0.21\end{array}$ & $\begin{array}{c}25.73 \pm \\
0.54 \mathrm{a}\end{array}$ & $\begin{array}{c}6.95 \pm \\
0.09\end{array}$ & $\begin{array}{c}11.75 \pm \\
0.11 \mathrm{a}\end{array}$ & $\begin{array}{l}19.76 \pm \\
0.03 \mathrm{~d}\end{array}$ & $\begin{array}{l}1.74 \pm \\
0.02 \mathrm{a}\end{array}$ & $\begin{array}{c}53.54 \pm \\
0.25 \mathrm{a} \\
\end{array}$ & $\begin{array}{l}2.71 \pm \\
0.03 \mathrm{~b}\end{array}$ & $\begin{array}{l}1.23 \pm \\
0.20 \mathrm{a}\end{array}$ \\
\hline 900 (C900) & 120 & $\begin{array}{c}6.04 \pm \\
0.21 \\
\end{array}$ & $\begin{array}{l}26.23 \pm \\
0.54 \mathrm{a} \\
\end{array}$ & $\begin{array}{l}7.22 \pm \\
0.09 \\
\end{array}$ & $\begin{array}{l}11.84 \pm \\
0.11 \mathrm{a} \\
\end{array}$ & $\begin{array}{r}20.20 \pm \\
0.03 \mathrm{a} \\
\end{array}$ & $\begin{array}{l}1.75 \pm \\
0.02 \mathrm{a} \\
\end{array}$ & $\begin{array}{r}53.45 \pm \\
0.25 \mathrm{a} \\
\end{array}$ & $\begin{array}{l}2.65 \pm \\
0.03 \mathrm{~b}\end{array}$ & $\begin{array}{l}1.26 \pm \\
0.20 \mathrm{a} \\
\end{array}$ \\
\hline $1200(\mathrm{C} 1200)$ & 120 & $\begin{array}{c}6.01 \pm \\
0.21\end{array}$ & $\begin{array}{c}25.89 \pm \\
0.54 \mathrm{a}\end{array}$ & $\begin{array}{l}7.15 \pm \\
0.09\end{array}$ & $\begin{array}{c}11.66 \pm \\
0.11 \mathrm{a} \\
\end{array}$ & $\begin{array}{l}19.88 \pm \\
0.03 \mathrm{c}\end{array}$ & $\begin{array}{l}1.75 \pm \\
0.02 \mathrm{a}\end{array}$ & $\begin{array}{c}54.12 \pm \\
0.25 \mathrm{a} \\
\end{array}$ & $\begin{array}{l}2.72 \pm \\
0.03 \mathrm{~b}\end{array}$ & $\begin{array}{l}1.22 \pm \\
0.20 \mathrm{a}\end{array}$ \\
\hline \multicolumn{11}{|c|}{ Fat $\times$ L-carnitine } \\
\hline F5 C0 & 60 & $\begin{array}{c}5.84 \pm \\
0.29 \\
\end{array}$ & $\begin{array}{l}19.60 \pm \\
0.77 \mathrm{~b} \\
\end{array}$ & $\begin{array}{l}7.08 \pm \\
0.13 \\
\end{array}$ & $\begin{array}{l}10.75 \pm \\
0.15 \mathrm{c} \\
\end{array}$ & $\begin{array}{l}13.76 \pm \\
0.05 \mathrm{~g}\end{array}$ & $\begin{array}{l}1.45 \pm \\
0.03 \mathrm{~b} \\
\end{array}$ & $\begin{array}{l}43.32 \pm \\
0.36 \mathrm{~b} \\
\end{array}$ & $\begin{array}{l}3.15 \pm \\
0.05 \mathrm{a} \\
\end{array}$ & $\begin{array}{l}1.05 \pm \\
0.29 \mathrm{~b} \\
\end{array}$ \\
\hline F5 C300 & 60 & $\begin{array}{c}5.88 \pm \\
0.29\end{array}$ & $\begin{array}{c}25.48 \pm \\
0.77 \mathrm{a}\end{array}$ & $\begin{array}{l}7.17 \pm \\
0.13\end{array}$ & $\begin{array}{l}11.62 \pm \\
0.15 \mathrm{~b}\end{array}$ & $\begin{array}{l}19.66 \pm \\
0.05 \mathrm{~d}\end{array}$ & $\begin{array}{l}1.75 \pm \\
0.03 \mathrm{a}\end{array}$ & $\begin{array}{c}52.33 \pm \\
0.36 \mathrm{a}\end{array}$ & $\begin{array}{l}2.66 \pm \\
0.05 \mathrm{~b}\end{array}$ & $\begin{array}{l}1.25 \pm \\
0.29 \mathrm{a}\end{array}$ \\
\hline F5 C600 & 60 & $\begin{array}{c}5.91 \pm \\
0.29\end{array}$ & $\begin{array}{c}25.23 \pm \\
0.77 \mathrm{a}\end{array}$ & $\begin{array}{c}6.99 \pm \\
0.13\end{array}$ & $\begin{array}{l}11.69 \pm \\
0.15 \mathrm{~b}\end{array}$ & $\begin{array}{c}19.32 \pm \\
0.05 \mathrm{e}\end{array}$ & $\begin{array}{l}1.73 \pm \\
0.03 \mathrm{a}\end{array}$ & $\begin{array}{c}52.74 \pm \\
0.36 \mathrm{a}\end{array}$ & $\begin{array}{l}2.73 \pm \\
0.05 \mathrm{~b}\end{array}$ & $\begin{array}{l}1.22 \pm \\
0.29 \mathrm{a}\end{array}$ \\
\hline F5 C900 & 60 & $\begin{array}{c}5.95 \pm \\
0.29\end{array}$ & $\begin{array}{c}26.03 \pm \\
0.77 \mathrm{a} \\
\end{array}$ & $\begin{array}{c}7.27 \pm \\
0.13\end{array}$ & $\begin{array}{l}11.73 \pm \\
0.15 b\end{array}$ & $\begin{array}{l}20.08 \pm \\
0.05 b c\end{array}$ & $\begin{array}{l}1.76 \pm \\
0.03 \mathrm{a}\end{array}$ & $\begin{array}{c}53.00 \pm \\
0.36 \mathrm{a} \\
\end{array}$ & $\begin{array}{l}2.64 \pm \\
0.05 b\end{array}$ & $\begin{array}{l}1.26 \pm \\
0.29 \mathrm{a} \\
\end{array}$ \\
\hline F5 C1200 & 60 & $\begin{array}{c}5.90 \pm \\
0.29\end{array}$ & $\begin{array}{c}25.31 \pm \\
0.77 \mathrm{a}\end{array}$ & $\begin{array}{c}7.20 \pm \\
0.13\end{array}$ & $\begin{array}{l}11.53 \pm \\
0.15 \mathrm{~b}\end{array}$ & $\begin{array}{c}19.41 \pm \\
0.05 \mathrm{e}\end{array}$ & $\begin{array}{l}1.74 \pm \\
0.03 \mathrm{a}\end{array}$ & $\begin{array}{c}53.89 \pm \\
0.36 \mathrm{a}\end{array}$ & $\begin{array}{l}2.78 \pm \\
0.05 \mathrm{~b}\end{array}$ & $\begin{array}{l}1.19 \pm \\
0.29 \mathrm{a}\end{array}$ \\
\hline F10 C0 & 60 & $\begin{array}{c}5.78 \pm \\
0.29 \\
\end{array}$ & $\begin{array}{c}20.05 \pm \\
0.77 \mathrm{~b}\end{array}$ & $\begin{array}{c}7.15 \pm \\
0.13 \\
\end{array}$ & $\begin{array}{r}10.54 \pm \\
0.15 \mathrm{c}\end{array}$ & $\begin{array}{c}14.27 \pm \\
0.05 \mathrm{f}\end{array}$ & $\begin{array}{r}1.49 \pm \\
0.03 \mathrm{~b}\end{array}$ & $\begin{array}{c}43.96 \pm \\
0.36 \mathrm{~b}\end{array}$ & $\begin{array}{l}3.08 \pm \\
0.05 \mathrm{a}\end{array}$ & $\begin{array}{r}1.08 \pm \\
0.29 \mathrm{~b}\end{array}$ \\
\hline F10 C300 & 60 & $\begin{array}{c}6.07 \pm \\
0.29 \\
\end{array}$ & $\begin{array}{c}25.81 \pm \\
0.77 \mathrm{a} \\
\end{array}$ & $\begin{array}{c}7.15 \pm \\
0.13\end{array}$ & $\begin{array}{c}12.35 \pm \\
0.15 \mathrm{a}\end{array}$ & $\begin{array}{l}19.74 \pm \\
0.05 \mathrm{~d}\end{array}$ & $\begin{array}{l}1.73 \pm \\
0.03 \mathrm{a}\end{array}$ & $\begin{array}{c}54.45 \pm \\
0.36 \mathrm{a} \\
\end{array}$ & $\begin{array}{l}2.76 \pm \\
0.05 b\end{array}$ & $\begin{array}{l}1.21 \pm \\
0.29 \mathrm{a}\end{array}$ \\
\hline F10 C600 & 60 & $\begin{array}{c}6.04 \pm \\
0.29\end{array}$ & $\begin{array}{c}26.23 \pm \\
0.77 \mathrm{a}\end{array}$ & $\begin{array}{c}6.90 \pm \\
0.13\end{array}$ & $\begin{array}{c}11.81 \pm \\
0.15 \mathrm{~b}\end{array}$ & $\begin{array}{l}20.20 \pm \\
0.05 \mathrm{ab}\end{array}$ & $\begin{array}{c}1.74 \pm \\
0.03 \mathrm{a}\end{array}$ & $\begin{array}{c}54.34 \pm \\
0.36 \mathrm{a}\end{array}$ & $\begin{array}{c}2.69 \pm \\
0.05 \mathrm{~b}\end{array}$ & $\begin{array}{c}1.23 \pm \\
0.29 \mathrm{a}\end{array}$ \\
\hline F10 C900 & 60 & $\begin{array}{c}6.13 \pm \\
0.29 \\
\end{array}$ & $\begin{array}{c}26.44 \pm \\
0.77 \mathrm{a} \\
\end{array}$ & $\begin{array}{c}7.16 \pm \\
0.13\end{array}$ & $\begin{array}{l}11.94 \pm \\
0.15 \mathrm{ab}\end{array}$ & $\begin{array}{c}20.31 \pm \\
0.05 \mathrm{a}\end{array}$ & $\begin{array}{l}1.74 \pm \\
0.03 \mathrm{a}\end{array}$ & $\begin{array}{c}53.89 \pm \\
0.36 \mathrm{a} \\
\end{array}$ & $\begin{array}{l}2.65 \pm \\
0.05 \mathrm{~b}\end{array}$ & $\begin{array}{l}1.25 \pm \\
0.29 \mathrm{a}\end{array}$ \\
\hline F10 C1200 & 60 & $\begin{array}{c}6.12 \pm \\
0.29 \\
\end{array}$ & $\begin{array}{c}26.46 \pm \\
0.77 \mathrm{a}\end{array}$ & $\begin{array}{c}7.11 \pm \\
0.13\end{array}$ & $\begin{array}{c}11.79 \pm \\
0.15 b\end{array}$ & $\begin{array}{c}20.34 \pm \\
0.05 \mathrm{a}\end{array}$ & $\begin{array}{c}1.75 \pm \\
0.03 \mathrm{a}\end{array}$ & $\begin{array}{c}54.34 \pm \\
0.36 \mathrm{a}\end{array}$ & $\begin{array}{c}2.67 \pm \\
0.05 \mathrm{~b}\end{array}$ & $\begin{array}{r}1.24 \pm \\
0.29 \mathrm{a}\end{array}$ \\
\hline
\end{tabular}

Means followed by the same letter in each column for each factor are not significantly different $(\mathrm{P}<0.05)$. 
The present study revealed that, L-carnitine caused a significant increase in BW, WG and SGR of Nile tilapia, O. niloticus and these results in accordance with those observed by Jayaprakas et al., (1996) and Azab et al., (2002) who found that, supplementation of L-carnitine to Nile tilapia diets significantly increases WG and improves FCR. In the same respect, Jayaprakas and Sambhu (1998) found significant increase in growth of Pearlspot, Etroplus suratensi when treated with $750 \mathrm{mg}$ carnitine/kg diet.

With regard to the interaction between dietary lipid and L-carnitine, results of Table 2 indicated that, within each lipid level; the lower L-carnitine level $(300 \mathrm{mg} / \mathrm{kg}$ diet) improved significantly BW, BL, WG, SGR, FI, FCR and PER but did not significantly different from the higher L-carnitine levels $(600,900$ and $1200 \mathrm{mg} / \mathrm{kg}$ diet). The lack of a strong L-carnitine effect on tilapia growth at the higher levels $(600,900$ or $1200 \mathrm{mg} / \mathrm{kg} \mathrm{diet})$ in the present experiment may due to the ability of fish to synthesize adequate quantities of L-carnitine for lipid metabolism. The diets were limited in lysine or methionines which are precursors for L-carnitine synthesis. If a limited precursor pool was available for metabolism, the higher L-carnitine levels may have had more dramatic influences on growth and/or proximate composition of gain. Azab et al., (2002) found that, growth rate of Nile tilapia improved when Lcarnitine was supplemented at a level of $900 \mathrm{mg} / \mathrm{kg}$ diet. However, Kheyyali and Tahari (1998) found that, L-carnitine supplementation to the level $250 \mathrm{mg} / \mathrm{kg}$ improved weight gain of rainbow trout, Oncorhynchus mykiss to $83 \%$ of the control and further supplementation of L-carnitine gave lower performance and $100 \mathrm{mg} \mathrm{L}$ carnitine/kg diet resulted in the lowest gain with $61 \%$ of the control.

\section{II-Carcass traits and body composition of Nile tilapia:}

Results of Table 3 indicated that, dietary lipids (5 and 10\%) had no significant effect on carcass traits of Nile tilapia. Compared to control all L-carnitine levels significantly increase the percentage of dress-out and flesh while the percentage of by-products significantly $(\mathrm{P}<0.05)$ decreased. With regard to the effect of interaction between dietary lipid and Lcarnitine, results of Table 3 revealed that, the diet contained 10\% lipid and 0 L-carnitine (F10 $\mathrm{C} 0$ ) recorded the lowest dress-out and flesh and the higher by-products percentages.

Table 3: Least square means and standard error for the effect of dietary fat and L-carnitine on carcass analysis of Nile tilapia.

\begin{tabular}{|c|c|c|c|c|}
\hline Item & No. & Dress-out $\%$ & Flesh \% & By-products $\%$ \\
\hline \multicolumn{5}{|l|}{ Fat $(\mathbf{F})$} \\
\hline $5 \%(\mathrm{~F} 5)$ & 30 & $49.95 \pm 0.42$ & $34.71 \pm 0.48$ & $57.44 \pm 0.48$ \\
\hline $10 \%(\mathrm{~F} 10)$ & 30 & $49.06 \pm 0.42$ & $34.77 \pm 0.48$ & $56.31 \pm 0.48$ \\
\hline \multicolumn{5}{|c|}{ L-carnitine mg/kg diet ( C ) } \\
\hline $0(\mathrm{C} 0)$ & 6 & $47.54 \pm 0.73 \mathrm{~b}$ & $32.95 \pm 0.83 \mathrm{~b}$ & $59.16 \pm 0.84 \mathrm{a}$ \\
\hline $300(\mathrm{C} 300)$ & 6 & $49.84 \pm 0.73 \mathrm{a}$ & $36.22 \pm 0.83 \mathrm{a}$ & $55.53 \pm 0.84 \mathrm{~b}$ \\
\hline $600(\mathrm{C} 600)$ & 6 & $49.45 \pm 0.73 \mathrm{a}$ & $35.96 \pm 0.83 \mathrm{a}$ & $55.37 \pm 0.84 \mathrm{~b}$ \\
\hline $900(\mathrm{C} 900)$ & 6 & $49.64 \pm 0.73 \mathrm{a}$ & $33.91 \pm 0.83 \mathrm{a}$ & $56.96 \pm 0.84 \mathrm{~b}$ \\
\hline $1200(\mathrm{C} 1200)$ & 6 & $51.06 \pm 0.73 \mathrm{a}$ & $35.27 \pm 0.83 \mathrm{a}$ & $56.82 \pm 0.84 \mathrm{~b}$ \\
\hline \multicolumn{5}{|c|}{ Fat $\times$ L-carnitine } \\
\hline F5 C0 & 3 & $49.33 \pm 1.04 \mathrm{a}$ & $34.96 \pm 1.17 \mathrm{ab}$ & $57.31 \pm 1.19 \mathrm{ab}$ \\
\hline F5 C300 & 3 & $50.29 \pm 1.04 \mathrm{a}$ & $34.65 \pm 1.17 \mathrm{ab}$ & $57.57 \pm 1.19 \mathrm{ab}$ \\
\hline F5 C600 & 3 & $48.84 \pm 1.04 \mathrm{a}$ & $35.68 \pm 1.17 \mathrm{ab}$ & $57.34 \pm 1.19 \mathrm{ab}$ \\
\hline F5 C900 & 3 & $49.50 \pm 1.04 \mathrm{a}$ & $34.95 \pm 1.17 \mathrm{ab}$ & $57.48 \pm 1.19 \mathrm{ab}$ \\
\hline F5 C1200 & 3 & $51.43 \pm 1.04 \mathrm{a}$ & $35.18 \pm 1.17 \mathrm{ab}$ & $56.26 \pm 1.19 \mathrm{bc}$ \\
\hline F10 C0 & 3 & $45.75 \pm 1.04 \mathrm{~b}$ & $30.95 \pm 1.17 \mathrm{c}$ & $61.02 \pm 1.19 \mathrm{a}$ \\
\hline F10 C300 & 3 & $49.38 \pm 1.04 \mathrm{a}$ & $37.78 \pm 1.17 \mathrm{a}$ & $53.49 \pm 1.19 \mathrm{c}$ \\
\hline F10 C600 & 3 & $50.06 \pm 1.04 \mathrm{a}$ & $36.24 \pm 1.17 \mathrm{ab}$ & $53.39 \pm 1.19 \mathrm{c}$ \\
\hline F10 C900 & 3 & $49.79 \pm 1.04 \mathrm{a}$ & $32.86 \pm 1.17 \mathrm{bc}$ & $58.45 \pm 1.19 \mathrm{ab}$ \\
\hline F10 C1200 & 3 & $50.70 \pm 1.04 \mathrm{a}$ & $35.36 \pm 1.17 \mathrm{ab}$ & $57.38 \pm 1.19 \mathrm{ab}$ \\
\hline
\end{tabular}

Means followed by the same letter in each column for each factor are not significantly different $(\mathrm{P}<0.05)$. 
Proximate analysis of tilapia fish as affected by dietary lipid and L-carnitine outlined in Table 4. As described in this table, the lower lipid level $(5 \%)$ released the higher $(\mathrm{P}<0.05)$ moisture and ash and the lower fat content. Also it was found that, as dietary L-carnitine increased fat content of tilapia fish significantly $(\mathrm{P}<0.05)$ decreased and protein content significantly increased, while moisture and ash content did not significantly affected. The higher protein content and lower fat content were recorded with fish fed the diet contained the higher L-carnitine level $(1200 \mathrm{mg} / \mathrm{kg}$ diet).

With regard to the effect of interaction between dietary lipid and L-carnitine, results of Table 4 show that, within each dietary lipid level, increased L-carnitine level from 0 to $1200 \mathrm{mg} / \mathrm{kg}$ diet significantly decreased fat and relatively increased protein content of tilapia fish while moisture and ash had no clear trend. These results similar to those obtained by Jayaprakas and Sambhu (1998), they found that, body protein content increased while body lipid decreased $(\mathrm{P}<0.01)$ with carnitine administration to pearlspot, Ertoplus suratensis. In contrast with our results, some authors found that, dietary L-carnitine did not alter tissue composition of hybrid tilapia (Becker et al., 1999) and Nile tilapia, O. niloticus (Azab et al., 2002).

From these results, it can be concluded that, L-carnitine at concentrations ranged from 300 to $1200 \mathrm{mg} / \mathrm{kg}$ diet in dietary lipid 5 or $10 \%$ improve growth performance, feed efficiency, carcass traits and increase tissue protein and decrease tissue fat percentages of Nile tilapia.

Table 4: Least square means and standard error for the effect of dietary fat and L-carnitine on proximate analysis of tilapia fish.

\begin{tabular}{|l|l|l|l|l|l|}
\hline Item & No. & Moisture $\%$ & Protein \% & Fat \% & Ash \% \\
\hline Fat (F) & 30 & $76.18 \pm 0.25 \mathrm{a}$ & $69.79 \pm 0.20$ & $12.92 \pm 0.06 \mathrm{~b}$ & $17.04 \pm 0.06 \mathrm{a}$ \\
\hline $5 \%($ F5) & 30 & $74.40 \pm 0.25 \mathrm{~b}$ & $69.23 \pm 0.20$ & $14.47 \pm 0.06 \mathrm{a}$ & $13.83 \pm 0.06 \mathrm{~b}$ \\
\hline $10 \%$ (F10) & 6 & $75.09 \pm 0.44$ & $68.21 \pm 0.35 \mathrm{~b}$ & $14.78 \pm 0.10 \mathrm{a}$ & $15.20 \pm 0.11$ \\
\hline L-carnitine mg/kg diet ( C) \\
\hline 0 (C0) & 6 & $75.92 \pm 0.44$ & $69.06 \pm 0.35 \mathrm{ab}$ & $14.37 \pm 0.10 \mathrm{a}$ & $15.80 \pm 0.11$ \\
\hline $300($ C300) & 6 & $75.27 \pm 0.44$ & $68.91 \pm 0.35 \mathrm{~b}$ & $13.92 \pm 0.10 \mathrm{ab}$ & $15.28 \pm 0.11$ \\
\hline $600($ C600) & 6 & $74.68 \pm 0.44$ & $69.74 \pm 0.35 \mathrm{ab}$ & $13.50 \pm 0.10 \mathrm{ab}$ & $15.31 \pm 0.11$ \\
\hline 900 (C900) & 6 & $75.60 \pm 0.44$ & $70.31 \pm 0.35 \mathrm{a}$ & $12.89 \pm 0.10 \mathrm{~b}$ & $15.76 \pm 0.11$ \\
\hline $1200($ C1200) & 3 & $76.12 \pm 0.62 \mathrm{ab}$ & $67.71 \pm 0.50 \mathrm{e}$ & $13.57 \pm 1.14 \mathrm{~d}$ & $17.40 \pm 0.15 \mathrm{a}$ \\
\hline Fat $\times$ L-carnitine & 3 & $76.84 \pm 0.62 \mathrm{a}$ & $69.10 \pm 0.50 \mathrm{bc}$ & $13.13 \pm 1.14 \mathrm{e}$ & $17.82 \pm 0.15 \mathrm{a}$ \\
\hline F5 C0 & 3 & $75.87 \pm 0.62 \mathrm{ab}$ & $69.75 \pm 0.50 \mathrm{bc}$ & $12.88 \pm 1.14 \mathrm{e}$ & $16.88 \pm 0.15 \mathrm{~b}$ \\
\hline F5 C300 & 3 & $75.29 \pm 0.62 \mathrm{abc}$ & $69.96 \pm 0.50 \mathrm{bc}$ & $12.68 \pm 1.14 \mathrm{e}$ & $16.61 \pm 0.15 \mathrm{~b}$ \\
\hline F5 C600 & 3 & $75.99 \pm 0.62 \mathrm{ab}$ & $70.62 \pm 0.50 \mathrm{ab}$ & $12.68 \pm 1.14 \mathrm{e}$ & $16.64 \pm 0.15 \mathrm{~b}$ \\
\hline F5 C900 & 3 & $74.06 \pm 0.62 \mathrm{bc}$ & $68.71 \pm 0.50 \mathrm{~cd}$ & $15.99 \pm 1.14 \mathrm{a}$ & $13.00 \pm 0.15 \mathrm{e}$ \\
\hline F5 C1200 & 3 & $75.01 \pm 0.62 \mathrm{abc}$ & $69.03 \pm 0.50 \mathrm{bc}$ & $15.62 \pm 1.14 \mathrm{a}$ & $13.70 \pm 0.15 \mathrm{~d}$ \\
\hline F10 C0 & 3 & $74.67 \pm 0.62 \mathrm{bc}$ & $68.06 \pm 0.50 \mathrm{de}$ & $14.95 \pm 1.14 \mathrm{~b}$ & $13.67 \pm 0.15 \mathrm{~d}$ \\
\hline F10 C300 & 3 & $74.07 \pm 0.62 \mathrm{bc}$ & $69.52 \pm 0.50 \mathrm{bc}$ & $14.31 \pm 1.14 \mathrm{c}$ & $14.01 \pm 0.15 \mathrm{~d}$ \\
\hline F10 C600 & 3 & $75.20 \pm 0.62 \mathrm{abc}$ & $70.00 \pm 0.50 \mathrm{bc}$ & $13.11 \pm 1.14 \mathrm{e}$ & $14.88 \pm 0.15 \mathrm{c}$ \\
\hline F10 C900
\end{tabular}

Means followed by the same letter in each column for each factor are not significantly different $(\mathrm{P}<0.05)$

\section{Second experiment}

During the period between December to March 2004 water temperature in the experimental aquaria ranged between 9.35 and $11.50^{\circ} \mathrm{C}$ (Table 8). Final BW and BL of tilapia fish did not significantly affected by dietary L-carnitine. Results of cold tolerance challenge showed a significant advantage of all treatment groups which received the diets 
supplemented with L-carnitine compared to control group (had no L-carnitine supplementation). This was evident by the high survival rate for all L-carnitine supplemented groups. Fish group received L-carnitine at a level of 900 or $1200 \mathrm{mg} / \mathrm{kg}$ diet exhibited the best survival rate. Similar results were obtained by Harpaz et al., (1999). They found that, addition of L-carnitine to diets of Pelvicachromis pulcher at a level of 900 or $1000 \mathrm{mg} / \mathrm{kg}$ diet seems to yield the best protection against exposure to cold. Also, Soltan et al., (2015) found that, supplementation of O. niloticus with 350 $\mathrm{mg} / \mathrm{kg}$ diet L-carnitine significantly increased survival rate from $16 \%$ (control group) to $73 \%$ for treated fish.

The major goal in the over-wintering of tilapia fingerlings is to obtain a high survival rate of the fish and to keep them in good condition for future growth in the production ponds (Bakeer et al., 2005). Crab et al., (2009) demonstrated that, temperature in the covered ponds with polyethylene sheets could easily be controlled and was $0.4-4.9^{\circ} \mathrm{C}$ higher than the influent water. Hybrid tilapia fingerlings $(O$. niloticus $\times$ O. aureus) survival levels were excellent being $97 \pm 6 \%$ for $100 \mathrm{~g}$ fish and $80 \pm 4 \%$ for $50 \mathrm{~g}$ fish and these findings can help to overcome over-wintering problems, particularly mass mortality of fish due to low temperatures in the ponds. Therefore, the obtained results indicated the possibility of improving $O$. niloticus survival by supplementing tilapia diets with $350 \mathrm{mg} / \mathrm{kg}$ diet L-carnitine.

Table 5: Effect of L-carnitine levels in diets on cold tolerance of $O$. niloticus.

\begin{tabular}{|l|c|c|c|c|c|}
\hline \multirow{2}{*}{ Growth traits } & \multicolumn{5}{|c|}{ L-carnitine level } \\
\cline { 2 - 6 } & $0 \mathrm{mg} / \mathrm{kg}$ & $300 \mathrm{mg} / \mathrm{kg}$ & $600 \mathrm{mg} / \mathrm{kg}$ & $900 \mathrm{mg} / \mathrm{kg}$ & $1200 \mathrm{mg} / \mathrm{kg}$ \\
\hline Initial BW $(\mathrm{g})$ & $3.63 \pm 0.17$ & $3.70 \pm 0.17$ & $3.72 \pm 0.17$ & $3.71 \pm 0.17$ & $3.73 \pm 0.17$ \\
\hline Final BW $(\mathrm{g})$ & $7.51 \pm 0.76$ & $8.01 \pm 0.67$ & $8.36 \pm 0.67$ & $8.96 \pm 0.67$ & $9.01 \pm 0.65$ \\
\hline Initial BL(cm) & $6.58 \pm 0.08$ & $6.45 \pm 0.08$ & $6.37 \pm 0.08$ & $6.52 \pm 0.08$ & $6.58 \pm 0.08$ \\
\hline Final BL(cm) & $8.18 \pm 1.77$ & $8.01 \pm 1.57$ & $7.88 \pm 1.57$ & $7.81 \pm 1.51$ & $8.21 \pm 1.65$ \\
\hline Survival rate & $64.52 \pm 0.26 \mathrm{~d}$ & $75.81 \pm 0.26 \mathrm{c}$ & $82.26 \pm 0.26 \mathrm{~b}$ & $87.10 \pm 0.26 \mathrm{a}$ & $87.32 \pm 0.26 \mathrm{a}$ \\
\hline Temperature ${ }^{\circ} \mathbf{C}$ & 11.38 & 11.20 & 11.28 & 11.40 & 11.45 \\
\hline December & 10.42 & 10.51 & 11.50 & 10.91 & 11.30 \\
\hline January & 10.25 & 9.35 & 9.40 & 9.75 & 9.90 \\
\hline February & 10.70 & 10.40 & 10.60 & 10.70 & 10.90 \\
\hline Average ${ }^{\circ} \mathrm{C}$ & &
\end{tabular}

Means followed by the same letter in each column for each factor are not significantly different $(\mathrm{P}<0.05)$

\section{REFERENCES}

Abdel-Hakim, N. F.; Bakeer, M. N. and Soltan, M. A. (2001a). Effect of dietary protein levels on growth performance and pond productivity of Nile tilapia (Oreochromis niloticus), Eel (Anguilla anguilla) and Grey mullet (Mugil cephalus) reared in polyculture system. Egypt. J. Aquat. Biol. \& Fish., 5(4):6185.

Abdel-Hakim, N. F.; Hussein, M. S.; Bakeer, M. N. and Soltan, M. A. (2001 b). Effect of protein level and stocking density on growth performance of Nile tilapia (Oreochromis niloticus) cultured in tanks. Egyptian J. Nutrition and Feeds 4:763780.

AOAC (1990). Official Methods of Analysis. $15^{\text {th }}$ Ed. Published by the Association of Official Analytical Chemists. North Nineteen st. Auite 210 Arlingth, Virginia 2220/USA. 
Azab, M. E.; Fathalla, S. I. and Soltan, M. A. (2002). Effect of feed supplementation with L-carnitine on growth performance and body composition of Nile tilapia (Oreochromis niloticus). Suez Canal Vet. Med. J. 5(1):201-214 (special Issue).

Bakeer, M. N.; Soltan, M. A.; Tharwat, A. A. and Samra, I. M. (2005). Studies on overwintering of Nile tilapia (Oreochromis niloticus) fry. Annals of Agric. Sci., Moshtohor, 43(3):1067-1082.

Becker, K.; Scheiber, S.; Angoni, C. and Blum, R. (1999). Growth performance and feed utilization responded of Oreochromis niloticus $\times O$. aureus hybrids to Lcarnitine measured over a full fattening cycle under commercial conditions. Aquacult, 174: 313 - 322.

Bilinski, E. and Jonas, R. E. (1970). Effects of coenzyme A and carnitine on fatty acid oxidation by rainbow trout mitochondria. J. Fish. Res. Board Can., 27:857-864.

Chatzifotis, S.; Takeuchi, T. and Seikai, T., (1995). The effect of dietary L-carnitine on growth performance and lipid composition in red sea bream fingerlings. Fish. Sci. 61, 1004-1008.

Crab, R.; Kochva, M.; Verstraete, W. and Avnimelech, Y. (2009). Bio-flocs technology application in over-wintering of tilapia. Aquac, Eng 40:105-112.

Duncan, D. B. (1955). Multiple range and Multiple test. Biometerics, 11: 1-42.

Dunn, W. A. (1981). L-carnitine biosynthesis in vivo. J. Biol. Chem. 256, 1237-1244.

Harpaz, S.; Becker, K. and Blum, R. (1999). The effect of dietary L-carnitine supplementation on cold tolerance and growth of the ornamental Cichlid fish. J. Therm Biol, 24:57- 62.

Hassaan, M. S.; Wafa, M. A.; Soltan, M. A.; Goda, A. S. and Mogheeth, N. M. A. (2014a). Effect of Dietary Organic Salts on Growth, Nutrient Digestibility, Mineral Absorption and Some Biochemical Indices of Nile Tilapia; Oreochromis niloticus L. Fingerlings. World Appl. Sci. J., 29(1):47-55.

Hassaan, M. S.; Soltan, M. A. and Ghonemy, M. M. R. (2014b). Effect of synbiotics between Bacillus licheniformis and yeast extract on growth, hematological and biochemical indices of the Nile tilapia Oreochromis niloticus. The Egyptian J of Aqua Rese. 40:199-208.

Hassaan, M. S.; Soltan, M. A.; Agouz, H. M. and Badr, A. M. (2013). Influences of calcium/phosphorus ratio on supplemental microbial phytase efficiency for Nile tilapia (Oreochromis niloticus). Egyptian J of Aquat Rese, 39:205-213.

Jayaprakas, V. and Sambhu, C. (1998). Influence of dietary L-carnitine on growth and lipid metabolism in pearlspot, Etroplus suratensis (Bloch). Indian J. Exp. Biol., 36:1044-1048.

Jayaprakas, V.; Sambhu, C. and Kumar, S. S. (1996). Effect of dietary L-carnitine on growth and reproductive performance of male Oreochromis mossambicus (peters). Fish. Techonol. 33: 84-90.

Ji, H.; Bradiley, T. M. and Tremblay, G. C. (1996). Atlantic salmon (Salmo salar) fed L-carnitine exhibit altered intermediary metabolism and reduced tissue lipid, but no change in growth rate. J. Nutr., 126: 1937-1950.

Kheyyali, D. and Tahari, S. (1998). Effect of supplemental dietary carnitine on growth and body composition of the rainbow trout (Oncorthynchus mykiss). In L. Coetzee, J. Gon and C. Kulongowski (eds.). African Fishes and Fisheries Diversity and Utilization. Grahams town South Africa FIUSA, Paradi, pp. 214.

Lovell, R. T. (1981). Laboratory manual for fish feed analysis and fish nutrition studies. Auburn Univ., Alabama.

NRC (1993). National Research Council. Nutrient Requirements of Fish. National Academy Press, Washington, DC, 114 pp. 
Santulli. A.; Puccia, E. and D'Amelio, V. (1990). Preliminary study on the effect of short-term carnitine treatment on nucleic acid and protein metabolism in sea bass (Dicentrachus Labrax) fry. Aquacult, 87: 85-89.

SAS (1996). SAS Procedure Guide "version 6.12 Ed". SAS Institute Inc., Cary, NC, USA.

Soltan, M. A.; Abdella, M.M.; El-Sayaad, G.A. and Abu-El Wafa, M. H. (2015). Effect of some over-wintering regimes on survival and growth performance of Nile tilapia Oreochromis niloticus. Biological and Chem Rese., 2:362-374.

Soltan, M. A.; Mohamed, K. A. and Eid, A. H. (2006). Effect of protein to energy ratio on growth performance and body composition of red tilapia reared in freshwater. $\mathrm{J}$ the Egypt Aquacult Society, 1:57-68.

Soltan, M. A.; Radwan, A. A. and Samra, I. M. (2002). Effect of varying protein, energy and protein to energy ratio on growth, feed efficiency and body composition of Nile tilapia, Oreochromis niloticus. $1^{\text {st }}$ Annual Conference of the Egyptian Aquaculture Society, Al-Arish, North Sinai, Egypt, 13-15 December, 2002.

Torreele, E.; Van Der Sluiszen, A. and Verreth, J. (1993). The effect of dietary Lcarnitine on the growth performance in fingerlings of the African catfish (Clarias gariepinus) in relation to dietary lipid. Br. J. Nutr. 69: 289-299.

Tremblay, G. C. and Bradley, T. M. (1992). L-carnitine protect fish against acute ammonia toxicity. Comp. Biochem. Physiol. 101(C): 349-351.

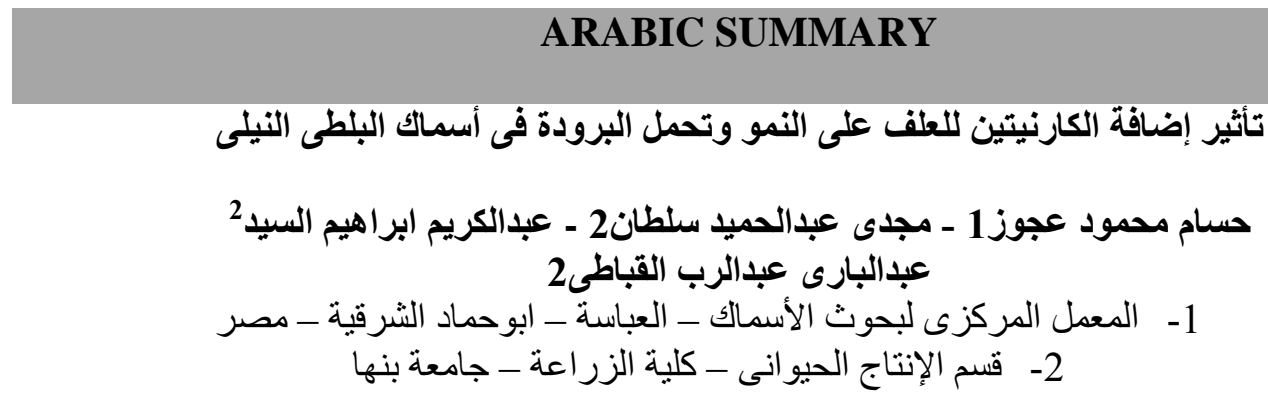

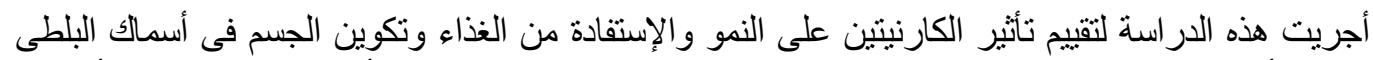

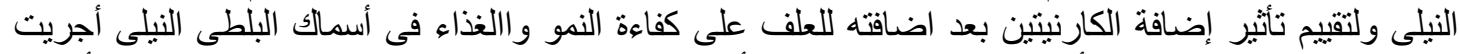

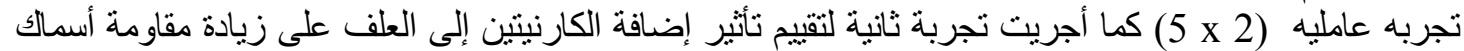

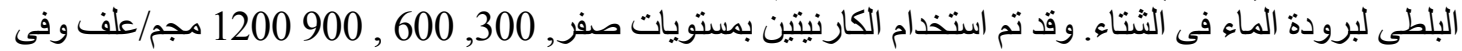

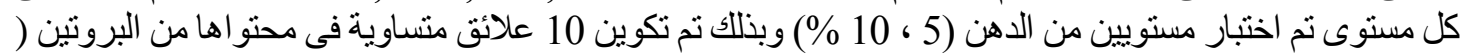

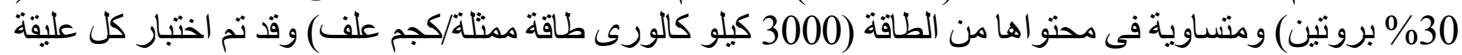

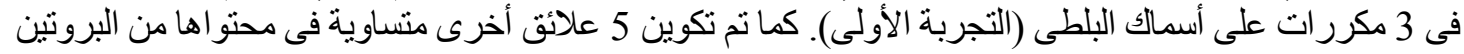

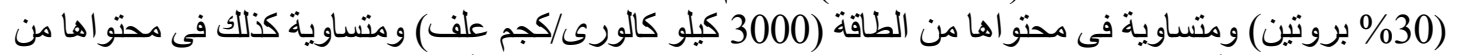

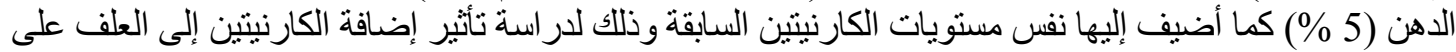

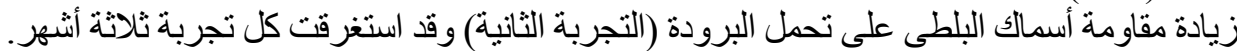

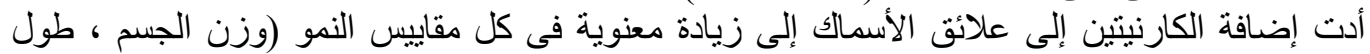

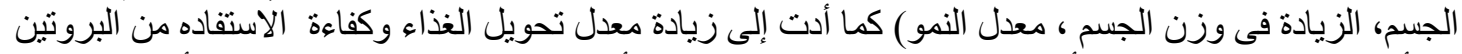

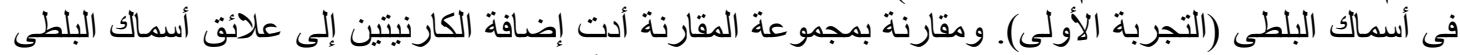

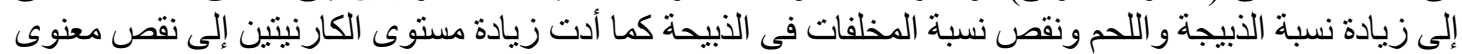

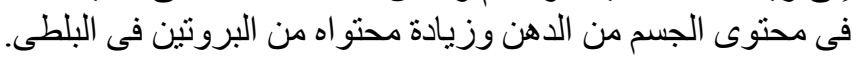

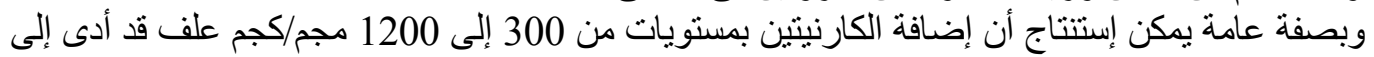

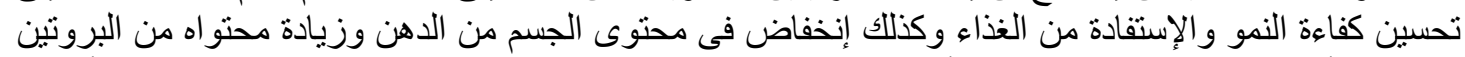
وذلك فى أسمالك البلطى و المبروك كما أدت إضافة الكارنيتين إلى علائق البلطى إلى إلى إلى زيادة نسبة الحيوية لأسماك

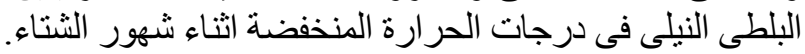

